\title{
Microhardness measurement in nonmetallic materials
}

\author{
V SARASWATI \\ Materials Development Laboratory. Indira Gandhi Center for Atomic Research, \\ Kalpakkam 603 102, India \\ MS received 11 May 1987; revised 3 September 1987
}

\begin{abstract}
The load dependence of Vicker's indentation hardness has been measured in some non-metallic materials and thin foils. A load of $100 \mathrm{~g}$ appears to be appropriate for comparing materials. Foil thickness, especially for soft metals like copper, must be at least twenty times the impression diagonal to get a correct value of hardness. The Meyer index varies for materials and can be used for comparison because it seems to pertain to the nature of chemical bonds. The index for boron carbide is similar to glass as was expected by Rice.
\end{abstract}

Keywords. Indentation hardness; microhardness; Vickers hardness; non-metallic materials.

\section{Introduction}

Hardness measurements are commonly carried out to estimate the mechanical strength of a material as it is a measure of resistance against plastic deformation. A microhardness tester using a Vickers or Knoops indenter is a compact machine which can give a quick and qualitative estimate of the hardness number. The resistance to penctration by a sharp indenter is the measure of hardness. Apart from being a strength microprobe it correlates other physical properties as well, such as the presence of other phases. inhomogeneity, anisotropy and surface conditions of a material (Gilman 1973). As the required size of material is small, it is a convenient method. But there are practical difficulties in its usage and comparison with results available in the literature, because the loads used are different. In the microhardness range ( $<1 \mathrm{~kg}$ load) the hardness number VHN is found 10 be a function of load applied, especially for non-metallic materials (Buckle 1959). In this work different non-metallic materials have been compared. Hard and brittle materials like the carbides, noncrystalline glasses, polymers and also metals have been used for measurements. Polycrystalline materials have been used to avoid anisotropy effects in comparison. It is felt that Meyer index can be used for comparing materials because it pertains to the nature of the chemical bond. Boron compounds, though crystalline, show amorphous-like behaviour (Golikova and Samatov 1983). The conduction mechanism in boron carbide was similar to amorphous semiconductors (Saraswati et al 1985). In this study the Meyer index of boron carbide is found to be similar to the random network glasses.

\section{Experiment}

The materials compared are sintered boron carbide, tungsten carbide, a metglass, rodalime glass. a plastic, araldite mould aluminum and copper foils to represent soft, hard materials. crystalline ceramics and non-crystalline glasses. The carbide pellets, in which the reflectivity was low, were polsshed with a diamond paste of $0.25 \mu \mathrm{m}$ grit 
size. Mechanical polishing affects the results. It was not adopted unless found necessary. The density of the tungsten carbide pellet was $98 \%$ of the theoretical density. The boron carbide pellets were $92 \%$ and $95 \%$ dense and the grain sizes were about $5 \mu$ and $10 \mu$ respectively. The metallic glass was a piece from a ribbon, $300 \mu \mathrm{m}$ thick. The copper foils were of thickness 400 and $1200 \mu \mathrm{m}$. The thin foil was $99.9 \%$ pure whereas the thick one was of commercial grade. The aluminum sheet was $2 \mathrm{~mm}$ thick. A $3 \mathrm{~mm}$ thick plastic piece and a well-aged araldite mould represent the polymers. The surface was ultrasonically cleaned with water for plastic and glass and swabbed with dilute acid for copper to remove the oxide layer. A Leitz microhardness tester with a diamond pyramidal indenter was used. The load could be varied from 5 to $500 \mathrm{~g}$ in fixed steps. The load duration could be varied. When the duration was changed from 10 to $50 \mathrm{sec}$ or more no appreciable change was detected. Hence it was kept at $25 \mathrm{sec}$ throughout the study. For each load an average of atleast ten impressions were recorded for measuring the length of impression diagonal to $d$. The hardness was calculated using the formula

$$
H=1854 \cdot 4\left(P / d^{2}\right),
$$

where $P$ is the load expressed in $\mathrm{g}, d$ is in microns and $H$ in $\mathrm{kg} / \mathrm{mm}^{2}$. Ridging and sinking of material on unloading result in impressions which are not square but are convex or concave (Buckle 1959). These affect the diagonal length measurements to an extent of $10 \%$. Errors also arise due to poor reflectivity, numerical aperture etc. When the impression is small, the percentage error is greater.

\section{Results and discussion}

\subsection{Influence of heterogeneities}

The influence of porosity and heterogeneities in the hardness values were noticed in the carbides as can be seen from figure 1 which shows a plot of hardness against the load applied in the carbides and a reference steel metal. The hardness is higher for a denser boron carbide. The humps seen for a boron carbide and tungsten carbide are attributed to impure phases present, which were noticed earlier (Saraswati 1987) in microstructure and radiation damage study. When a material contains precipitates or other heterogeneities the hardness can increase. This will create fluctuations in the hardness values and produce humps. Irradiation reduced the hardness further (Saraswati and Rao 1985). A load of 200 to $300 \mathrm{~g}$ produced a good impression. For smaller loads the impression was small and the measurement error was large whereas for loads of $500 \mathrm{~g}$ or more cracks appeared and reduced the hardness. Hardness variation due to load had also been observed earlier (Buckle 1959). In tungsten carbide cracks appeared above $200 \mathrm{~g}$ load.

\subsection{Surface and thickness effects}

In metglass $\left(\mathrm{Fe}_{40} \mathrm{Ni}_{40} \mathrm{~B}_{20}\right)$ and sodalime glass polishing was found to reduce the inhomogeneities and hardness value as denoted by the smoothened humps (figure 2). It is understandable if the heterogeneity was on the surface. As the glasses are quenched from the melt inhomogeneities are likely. In the metglass the hardness 


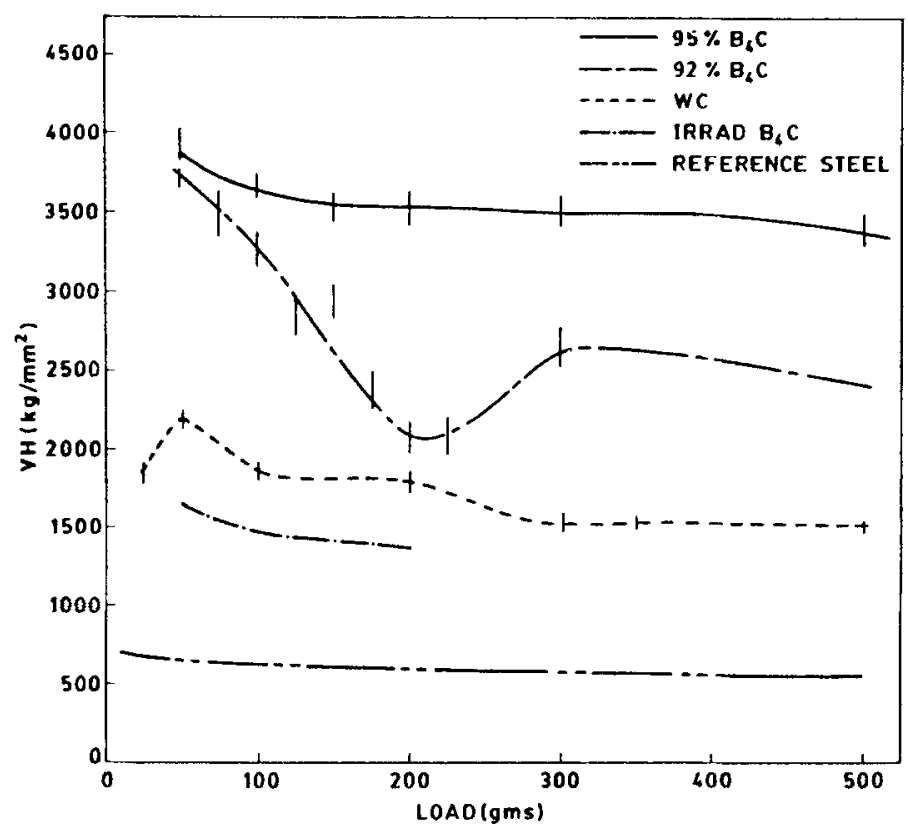

Figure 1. Hardness measurements in carbides.

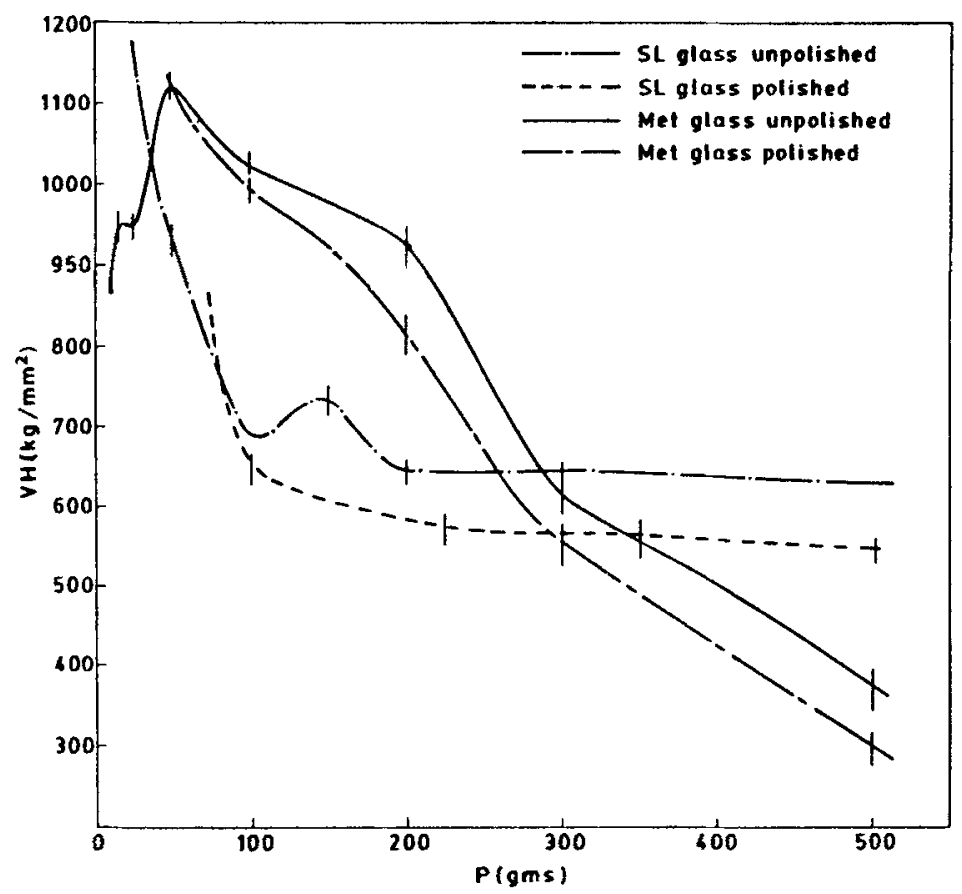

Figure 2. Eflect of polishing in glasses.

continued to decrease with increase in the load. In soda lime glass hardness approached a steady value above $100 \mathrm{~g}$. Comparison of a thin and thick foil showed the influence of thickness in copper. The specimen thickness normally recommended is 
about ten times the diagonal. At $15 \mathrm{~g}$ load, $d$ was $13 \mu \mathrm{m}$ for both the thin and the thick foil. At $25 \mathrm{~g}, d$ was $21 \mu \mathrm{m}$ for the thin foil which is about $1 / 20$ th the thickness of the foil but the hardness was lower. As the load increased the hardness number reduced rapidly. The impression was highly concave indicating severe sinking of the material. There was a visible depression in the foil as there was no resistance to deformation. Adhesion was also noticed. During plastic flow adhesion takes place in softer metals when the elastic stresses are smaller. In harder specimens elastic recovery breaks any such effect.

Thin foils of metals and alloys are normally used in some investigations because grinding these for TEM observations is easier. As is seen here the impression diagonal when compared to or is a large percentage of thickness erroneous results will be obtained unless the load is small. But very low loads such as 20 or $50 \mathrm{~g}$ can give an elevated value. The best suited load appears to be $100 \mathrm{~g}$ and hence materials investigated should have a reasonable thickness depending on the hardness of the material.

\subsection{Appropriate load}

At small loads i.e. $<50 \mathrm{~g}$, hardness increased rapidly in all materials. The exact load at which this happens depends on the material and normally appeared at impression diagonals less than $10 \mu \mathrm{m}$. This appeared to be due to surface, which might contain adsorbed gases or moisture or remnants of occluded abrasives or due to intrinsic difference in the surface and bulk density. One possibility is that equation (1) which

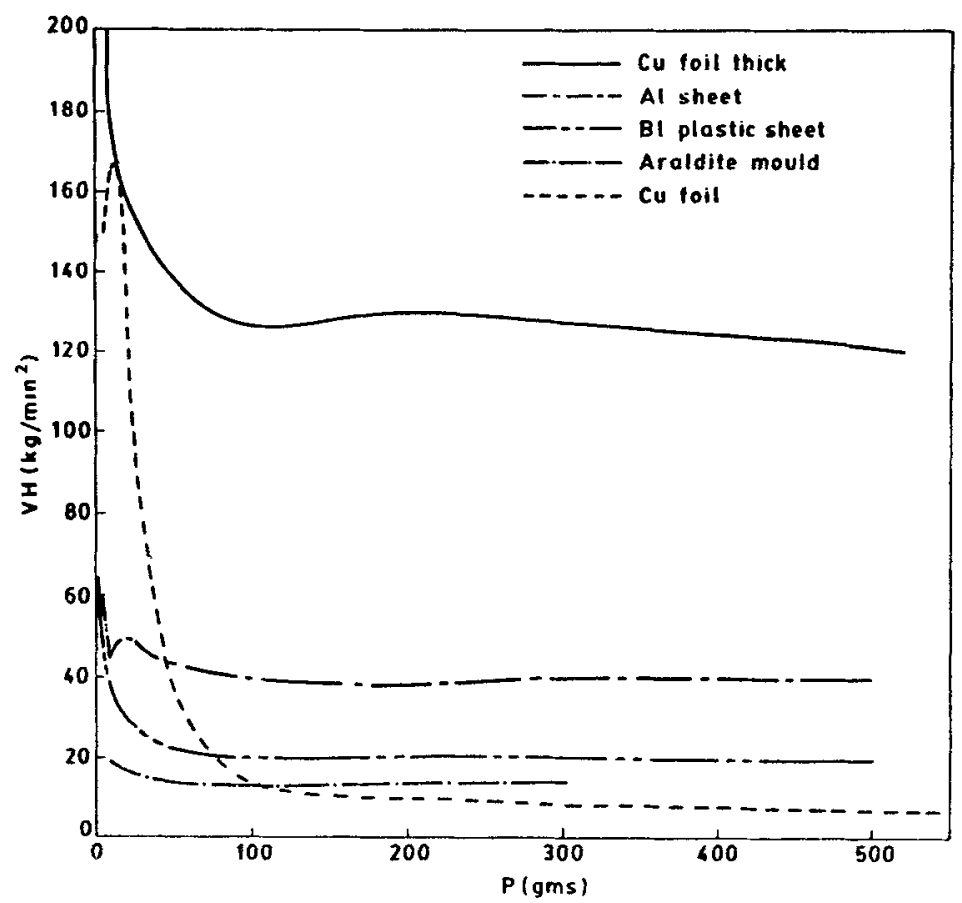

Figure 3. vickers hardness in polymers and copper forls. 
is derived for a plastic contact may not be valid at low loads when the contact is elastic. Several other explanations given which hold specially for metals were Orowan's model of workhardening and coherent regions suggested by Buckle (1973).

An empirical formula of Meyer states that load is related to impression diagonal as

$$
P=a d^{n}
$$

where $n$ is the Meyer index. Comparing with (1)

$$
H=b d^{n-2} \text {. }
$$

Thus hardness is independent of load only when $n=2$. From (2) we can see that a plot of $\log P$ vs $\log d$ should be a straight line with slope $n$. Figure 4 shows the plot for various materials investigated. The hard materials are on the left and the polymers are on the right in this diagram. The slopes deviate from 2 . The values are listed in table 1 . The metals have an index close to 2 , whereas the oxide glasses and ceramics have a low value of $n=1.66$. The metglass and polymers exhibit an intermediate value of 1.85 . The differing $n$ values had been observed earlier and attempts were made to modify (2) by incorporating other powers of $d$ in an empirical way. It is felt that $n$ is related to the chemical bond. In a metal, impurity and defects determine whether a material is soft or hard, whereas in a covalent compound the intrinsic strengths of the chemical bond determine its hardness. Gilman (1973) had shown that hardness vs elastic modulus had a slope of 1 for metals compared to $3 / 2$ for semiconductors and covalently-bonded materials. The difference arises because the How rate in metals is limited primarily by the interaction between dislocations, whereas in covalent crystals it is limited by the interaction between dislocation and chemical bonds in the compound i.e. short range (atomic) interactions are involved

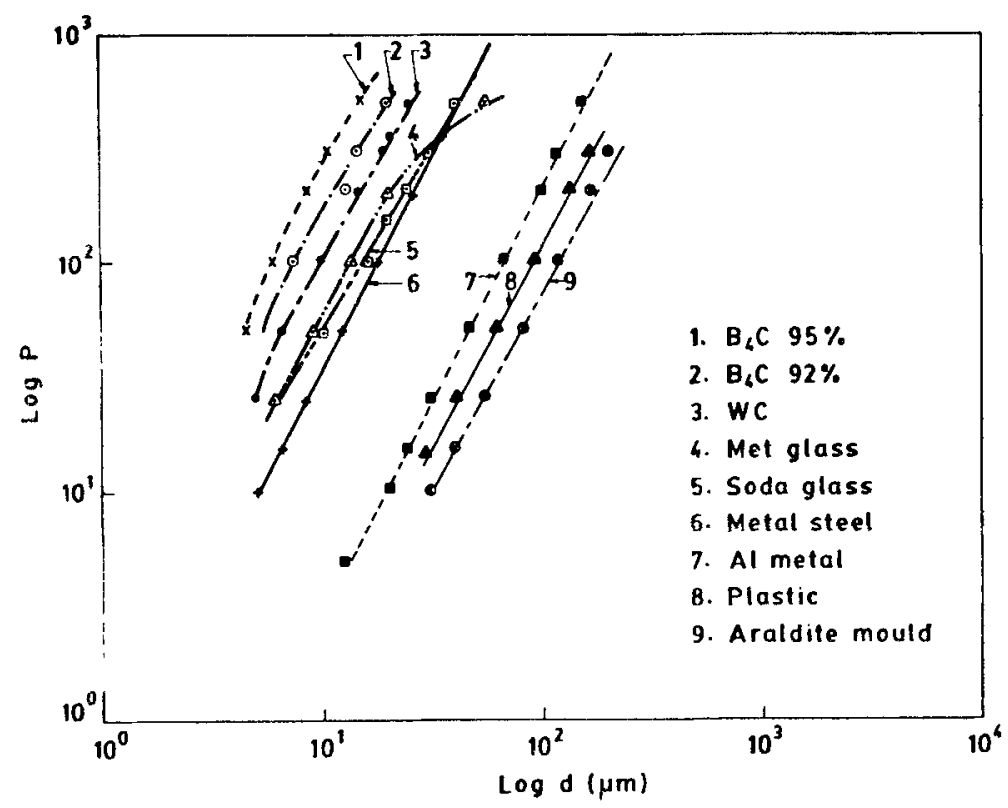

Figute f. forkd in diagonal in vallous materials. 
Table 1. Hardness at different loads and the slope of $\log P$ vs $\log d$.

\begin{tabular}{llrrrrr}
\hline Material & Thickness & HV 200* & HV 100† & HV 50 & HV(20) & Slope $n \pm 01$ \\
\hline $\mathrm{B}_{4} \mathrm{C} 95 \%$ & $2 \mathrm{~mm}$ & 3525 & 3650 & 3875 & & 1.68 \\
$\mathrm{~B}_{4} \mathrm{C} 92 \%$ & $2 \mathrm{~mm}$ & 3100 & 3250 & 3700 & & 1.66 \\
WC & $3 \mathrm{~mm}$ & 1800 & 1900 & 2200 & & 1.66 \\
Ref steel & $5 \mathrm{~mm}$ & 600 & 625 & 650 & & 1.96 \\
copper & $1200 \mu \mathrm{m}$ & 130 & 127 & 137 & $>200$ & \\
copper & $400 \mu \mathrm{m}$ & 10 & 14 & 37 & 167 & 1.92 \\
aluminum & $2 \mathrm{~mm}$ & 38 & 40 & 43 & $>50$ & 1.80 \\
plastic & $3 \mathrm{~mm}$ & 20 & 20 & 20 & $>60$ & 1.82 \\
araldite & $1 \mathrm{~cm}$ & 14 & 14 & 14 & 20 & 1.66 \\
sl glass polished & $2 \mathrm{~mm}$ & 570 & 650 & $>800$ & & 1.66 \\
sl glass & $2 \mathrm{~mm}$ & 640 & 680 & 930 & $>1000$ & 1.81 \\
unpolished & $300 \mu \mathrm{m}$ & 815 & 1000 & 1120 & & \\
Metglass & & & & & & \\
polished & $300 \mu \mathrm{m}$ & 925 & 1025 & 1110 & & \\
Metglass & & & & & \\
unpolished & & & & & & \\
\hline
\end{tabular}

${ }^{*} \mathrm{HVX}$ refers to hardness at a load $\mathrm{Xg}$.

†The deviation between HV 200 and HV 100 is the lowest.

in covalent materials whereas longer range (elastic) interactions are pertinent in metals. This is because the covalent bonds are highly stereospecific. Hence plastic characteristics will be closely connected with electronic characteristics of covalent materials. This can explain the difference in the Meyer index. It is also noted that the curves are linear in the load range 100 to $200 \mathrm{~g}$ even for plastics in which the hardness is low. The hardness values at different loads are compared in table 1 . If one considers a standard load for all materials, $100 \mathrm{~g}$ is appropriate except for hard carbides for which $200 \mathrm{~g}$ is probably better.

\subsection{Hardness of boron carbide}

A material accommodates indentation by slipping around the sides of the indenter or by compressing radially under the indenter. For ductile metals the microhardness $H$ is related to the uniaxial flow stress $Y$ as $H=C Y$ where $C$ is the constraint factor (Tabor 1951). For ductile metals $C=3$, whereas for polymers and glasses it is less than 3 . It is so because, in the region of indentation (influence zone), the plastic and elastic strains are comparable for non-metals whereas the strain is essentially plastic for ductile metals. An elastoplastic interpretation is needed when the material displaced by the indenter is accommodated elastically by the surrounding medium. This was taken into account by Marsh (1964) and Johnson (1970). Thus, materials can be distinguished as those with high $E / Y$ (e.g. metals) and those with low $E / Y$ (non-metals like glass or polymers) because of the following reasons: (i) metals are dense whereas the non-metals are not and so can be compressible under an indentation load, (ii) metals prefer deformation by slip whereas non-metals prefer compression and a uniform stress distribution as in liquids and (iii) metals normally have a long range order whereas order is short ranged in glasses and partial in polymers.

For glasses and polymers Marsh (1970) derived an expression

$$
H / Y=0.28+0.60 \ln 0.7 \mathrm{E} / Y \text {. }
$$


From this expression we get $E / Y=133$ when $H / Y=3$. This represents the critical value for distinguishing the low and high $E / Y$ regimes. Steels have $E / Y$ in the range 300 to 1000 depending upon the composition while glasses and polymers have $E / Y$ near 25. This variation is to be ascribed to their atomic structure and chemical bonds. The demarcating value for $H / E$ is 0.02 (metals have lower and non-metals higher). Many covalent ceramics are found to deform by slip or twinning like metals (Rice 1973) though $H / E$ is $>0.02$. Hence Rice (1973) suggested that low $E / Y$ materials should be further distinguished as those with low $E$ as for polymers and those with large $Y$ as for covalent crystalline ceramics like the carbides. $H / E$ is $0.07-$ 0.09 for boron carbide from experimental values. Table 1 shows that boron carbide is similar to glasses if the Meyer index is a criterion. Hence Rice's suggestion appears valid. Moreover several properties of boron carbide are akin to amorphous materials. It is a semiconductor with hopping electron conduction (Saraswati et al 1985) and has a temperature-dependent susceptibility (Saraswati and Paulose 1987).

Boron carbide has a hardness next only to diamond. Its structure consists of interconnected $B_{12}$ icosahedra in layers linked by C-B-C chains. Boron and carbon contain $p$ electrons in their outer orbitals and they are responsible for the high covalency of B-C bond. Hence sintering of this ceramic is difficult and is possible only close to the melting point, $2300^{\circ} \mathrm{C}$. Gilman (1973) has shown that for carbides a correlation between chemical bond and hardness can be expected because strong chemical forces inhibit dislocation movements. The heat of formation was found to be proportional to the hardness. According to Samsonov (1970), the high value of hardness in carbides is connected with the localization of valence electrons at nuclei with the formation of stable $\mathrm{sp}^{3}$ configuration which determines the rigidity and direction of chemical bonds. In the configuration-localization model of Samsonov (1970), the hardness of carbides is determined by the probability of stable $\mathrm{sp}^{3}$ configuration of valence electrons being formed at the $\mathrm{C}$ atoms and this depends on the donor-acceptor properties of the constituent metal. When a carbide is formed, the non-localized electrons of the metal are transferred to $\mathrm{C}$ atoms and are maintained there by the exchange interaction, stabilizing the $\mathrm{sp}^{3}$ configuration of $\mathrm{C}$. This $\mathrm{sp}^{3}$ configuration stability accounts for the hardness of boron carbide and diamond. Brittleness is also associated with the localization of electrons. Plastic deformation decreases with decreasing localization and increasing $\mathrm{sp}^{3}$ configuration stability. The high strength and melting point of boron carbide are attributed to this.

\section{Conclusions}

From the experimental observations it is concluded that (i) Vicker's microhardness numbers in non-metals increase at low loads independent of the surface finish and in all materials. At loads of $300 \mathrm{~g}$ or more, appearance of cracks reduces the hardness. A load of $100 \mathrm{~g}$ seems to be appropriate for comparing materials. For soft materials the specimen thickness should be atleast 15-20 times the diagonal impression length at this load. (ii) The metglasses normally come as ribbons, few hundred microns thick. The impression diagonal is small because the metglasses are hard. The inhomogeneity of the material can be tested using the load variation of hardness. (iii) The Meyer index varies for materials and can be used for comparison. Highly covalent boron carbide and sodalime glass have similar index probably because of the similar $\mathrm{sp}$ character of the bonds. 


\section{Acknowledgement}

The author thanks Dr P Rodriguez for his comments and suggestions.

\section{References}

Buckle H 1959 Metall. Rev. 449

Buckle H 1973 The science of hardness testing and its research applications (eds) J H Westbrook and H Conrad (Ohio: ASM) p. 453

Gilman J J 1973 The science of hardness testing and its research applications (eds) J H Westbrook and H Conrad (Ohio: ASM) p. 51

Golikova O A and Samatov H 1983 Phys. Status Solidi A 77449

Johnson K L 1970 J. Mech. Phys. Solids 18115

Marsh D M 1964 Proc. R. Soc. London A279 420

Rice R W 1973 The science of hardness testing and its research applications (eds) J H Westbrook and H Conrad (Ohio: ASM) p. 117

Samsonov G V 1970 Refractory carbides (New York: Consultants Bureau)

Saraswati V 1987 Nucl. Instrum. Methods B23 347

Saraswati V and Rao G V N 1985 J. Mater. Sci. Lett. 4260

Saraswati V and Paulose P L 1987 J. Less Common Metals 128185

Saraswati V, Nandedkar R V and Radhakrishnan T R 1985 Proc of Solid State Symp. Nagpur (Bombay: BARC) $\mathrm{C} 28123$

Tabor D 1951 The hardness of metals (Oxford; Clarendon Press) 